\title{
Generalized Homological Mirror Symmetry and Cubics
}

\author{
L. Katzarkov ${ }^{a}$ and V. Przyjalkowski ${ }^{b}$
}

Received September 2008

To the cherished memory

of our unforgettable teacher V.A. Iskovskikh

\begin{abstract}
We discuss an approach to studying Fano manifolds based on Homological Mirror Symmetry. We consider some classical examples from a new point of view.
\end{abstract}

DOI: $10.1134 /$ S0081543809010118

\section{INTRODUCTION}

An important part of the legacy of V.A. Iskovskikh is his work on rationality of algebraic varieties. So it makes perfect sense to dedicate to him a paper containing a discussion on the subject of rationality from a new perspective-from the point of view of Homological Mirror Symmetry (HMS).

We begin by introducing some geometric generalizations of Homological Mirror Symmetry. We give an idea for showing nonrationality of generic three- and four-dimensional cubics. We elaborate the following line of thought. First, in Section 3 we study the behavior of Landau-Ginzburg models under birational transformations (see Theorem 3.2). After that we suggest possible birational invariants - the monodromy acting on the transcendental part of the second cohomology of a generic fiber. This monodromy is equal to the Serre functor of the subcategory semiorthogonal to the exceptional objects in the semiorthogonal decomposition of the derived category of a nonsemisimple Fano variety. At the end of Section 4 we introduce a new stronger invariant-the monodromy of the perverse sheaf of vanishing cycles associated with a Landau-Ginzburg model. We stress that HMS should be seen more as a correspondence between superschemes and Kato stratified spaces. We briefly justify why these monodromies constitute birational invariants and carry out a concrete calculation in the case of a four-dimensional cubic.

Due to restrictions in time and space our discussion here is rather terse. We will refer heavily to the papers [7] and [6]. We promise to redeem ourselves providing more details in a future paper "Homological Mirror Symmetry, Monodromies and Cycles."

\section{GENERALIZED HMS}

Mirror symmetry was introduced as a duality between two $N=2$ superconformal field theories. Historically, the first version of the HMS conjecture was formulated for Calabi-Yau manifolds. Generalizations to symplectic manifolds with nonzero first Chern class and the role of LandauGinzburg models as their mirrors appeared soon afterward, first in mathematics and then in physics. From a mathematical point of view, these generalizations cover a variety of issues of vastly different complexity. We briefly discuss the classical HMS formulated by Kontsevich in Table 1 referring to [6] for more details.

\footnotetext{
${ }^{a}$ University of Miami, Coral Gables, FL 33124, USA.

${ }^{b}$ Steklov Institute of Mathematics, Russian Academy of Sciences, ul. Gubkina 8, Moscow, 119991 Russia.
} 
Table 1

\begin{tabular}{|c|c|}
\hline A-models (symplectic) & \multicolumn{1}{c|}{ B-models (algebraic) } \\
\hline$X=(X, \omega)$ : a closed symplectic manifold & $X:$ a smooth projective variety \\
$\begin{array}{l}\text { Fukaya category Fuk }(X): \\
\text { objects are Lagrangian submanifolds } L \\
\text { (equipped with flat line bundles); morphisms } \\
\text { are given by the Floer cohomology } \operatorname{HF}^{*}\left(L_{0}, L_{1}\right)\end{array}$ & $\begin{array}{l}\text { Derived category } D^{\mathrm{b}}(X) \text { : } \\
\text { objects are complexes of coherent sheaves } \mathcal{E} ; \\
\text { morphisms are } \operatorname{Ext}^{*}\left(\mathcal{E}_{0}, \mathcal{E}_{1}\right)\end{array}$ \\
\hline
\end{tabular}

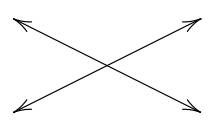

$Y$ : a noncompact symplectic manifold with a proper map $W: Y \rightarrow \mathbb{C}$ which is a symplectic fibration with singularities

Relative Fukaya category $\operatorname{Fuk}(W)$ : objects are Lagrangian submanifolds $L \subset Y$ which, at infinity, are fibered over $\mathbb{R}^{+} \subset \mathbb{C}$. The morphisms are $\operatorname{HF}^{*}\left(L_{0}^{+}, L_{1}\right)$, where the superscript + indicates a perturbation removing intersection points at infinity

$Y$ : a smooth quasiprojective variety with a proper holomorphic map $W: Y \rightarrow \mathbb{C}$

The category $D_{\text {sing }}^{\mathrm{b}}(W)$ of algebraic B-branes, obtained by considering the singular fibers $Y_{z}=W^{-1}(z)$, dividing $D^{\mathrm{b}}\left(Y_{z}\right)$ by the subcategory of perfect complexes $\operatorname{Perf}\left(Y_{z}\right)$, and then taking the direct sum over all such $z$

Table 2. HMS for Fano varieties

\begin{tabular}{|c|c|}
\hline B side & A side \\
$(X, D)$ & $\left(Y, Y_{\infty}, W\right)$ \\
\hline$D^{\mathrm{b}}(D)$ & $\operatorname{Fuk}\left(Y_{\infty}\right)$ \\
$i^{*} \mid i_{*}$ & \\
$D^{\mathrm{b}}(X)$ & $\operatorname{FS}\left|i^{*}\right| i_{*}$ \\
$\cup$ & $\cup$ \\
$D_{\text {compact }}^{\mathrm{b}}(X \backslash D)$ & $\operatorname{FS}_{\text {compact }}(Y, W)$ \\
support & Lagrangians \\
$D^{\mathrm{b}}(X \backslash D)$ & $\operatorname{Fuk}_{\text {wrapped }}\left(Y^{\prime}\right)$ \\
\hline
\end{tabular}

The smooth quasiprojective variety $Y$ with a proper holomorphic map $W: Y \rightarrow \mathbb{C}$ is often called a Landau-Ginzburg model or a Landau-Ginzburg mirror of $X$. Next we enhance HMS by supplementing the above setup with additional categorical and geometric structures.

In Table 2 we make HMS depend on additional geometric data: a divisor $D$ on the B side and a modified Landau-Ginzburg mirror on the A side. The wrapped Fukaya category appearing in Table 2 has its Lagrangians going many times around a given divisor vertical with respect to $W$, and in such a way it records an additional filtration. On the B side this corresponds to the log mixed Hodge structure associated with $X \backslash D$. These additional geometric data and categorical correspondences allow one in many cases to relate HMS to Hodge theory and draw geometric conclusions (see [6]). Finally, $Y_{\infty}$ is a marked smooth fiber different from the fiber at infinity of the Landau-Ginzburg model.

In the next section we employ these new settings in order to get geometric consequences. 
Table 3. Birational Geometry-HMS vocabulary

\begin{tabular}{|c|c|}
\hline Birational Geometry & Homological Mirror Symmetry \\
\hline$X$ & $w: Y \rightarrow \mathbb{C P}^{1}$ \\
Blow-up & Adding a singular fiber \\
Blow-down & Taking a singular fiber to $\infty$ \\
\hline
\end{tabular}

\section{APPLICATIONS TO BIRATIONAL GEOMETRY}

We consider some applications to Birational Geometry. First, in Table 3 we suggest a conjectural dictionary relating Birational Geometry and HMS. It is based on the following theorems:

Theorem 3.1 [9]. Let $X$ be a smooth projective variety and $X_{Y}$ be a blow-up of $X$ in a smooth subvariety $Y$ of codimension $k$. Then $D^{\mathrm{b}}\left(X_{Y}\right)$ has a semiorthogonal decomposition $\left(D^{\mathrm{b}}(X), D^{\mathrm{b}}(Y)_{k-1}, \ldots, D^{\mathrm{b}}(Y)_{1}\right)$. Here $D^{\mathrm{b}}(Y)_{i}$ are the corresponding twists by $\mathcal{O}(i)$.

This B-side statement has an A-model counterpart, which relates suitable noncompactified Landau-Ginzburg mirrors $\operatorname{LG}(X), \operatorname{LG}(Y)$, and $\operatorname{LG}\left(X_{Y}\right)$. In [1] the following result is discussed:

Theorem 3.2 [1]. The variety $X_{Y}$ has a Landau-Ginzburg mirror $\mathrm{LG}\left(X_{Y}\right)$ such that, for a suitable value of a constant $R>0$, the region $\{|W|<R\}$ is topologically equivalent to $\mathrm{LG}(X)$, while the region $\{|W|>R\}$ contains $k-1$ clusters of critical values each of which is topologically equivalent to a stabilization of $\mathrm{LG}(Y)$. In particular, $\mathbb{F}\left(\mathrm{LG}\left(X_{Y}\right)\right)$ admits a semiorthogonal decomposition $\left\langle\mathbb{F}(\mathrm{LG}(X)), \mathbb{F}(\mathrm{LG}(Y))_{k-1}, \ldots, \mathbb{F}(\mathrm{LG}(Y))_{1}\right\rangle$.

This theorem justifies Table 3 and suggests that studying the monodromy around singular fibers of Landau-Ginzburg models could yield this result. We proceed by exploring this idea on examples.

Our first example is a three-dimensional cubic. Applying the standard Hori-Vafa procedure, we get the following Landau-Ginzburg mirror:

$$
x y u v w=(u+v+w)^{3} \cdot t
$$

with potential $W=x+y$. Here $(u: v: w) \in \mathbb{C P}^{2},(x, y) \in \mathbb{A}^{2}$, and $t$ is a volume constant. The singular set over $W=0$ of the smooth compactification of this Landau-Ginzburg model looks as in Fig. 1.

The above Landau-Ginzburg model represents a family of K3 surfaces. The fiber over $W=0$ consists of six surfaces, and the monodromy around it is quasiunipotent.

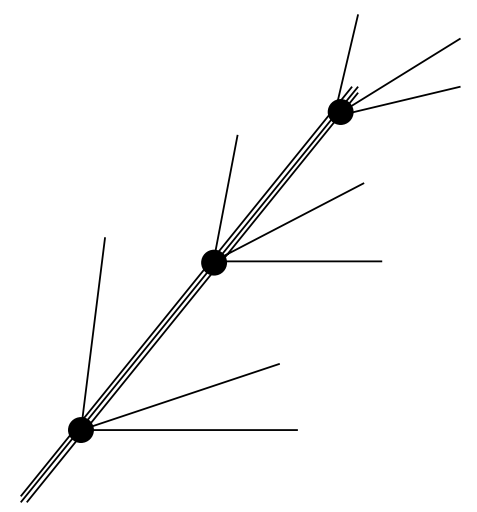

Fig. 1. The singular set for the LG of a cubic threefold. 
Table 4. Monodromies and rationality of Fano threefolds

\begin{tabular}{|c|c|c||c|c|c|}
\hline & $M$ & $R$ & & $M$ & $R$ \\
\hline $\mathbb{P}^{4} \supset X_{4}$ & $q$ & - & $\mathbb{P}^{3}$ & $u$ & + \\
$\mathbb{P}^{6} \supset Q_{1} \cap Q_{2} \cap Q_{3}$ & $q$ & - & $\mathbb{P}^{5} \supset Q_{1} \cap Q_{2}$ & $u$ & + \\
$V_{10}$ & $q$ & - & $V_{5}$ & $u$ & + \\
\hline
\end{tabular}

A comparison of the works of Iskovskikh [3] and Golyshev [2] implies as an immediate consequence the following:

Theorem 3.3 (Golyshev-Iskovskikh). Let $X$ be a three-dimensional Fano variety and $Y \rightarrow \mathbb{C}$ be its Landau-Ginzburg mirror. Suppose that there exists a singular fiber with nonisolated singularities in $Y \rightarrow \mathbb{C}$ such that the corresponding monodromy is not unipotent. Then $X$ is not rational. ${ }^{1}$

We summarize some examples in Table 4 . Here $R$ stays for rational, $M$ for monodromy, $q$ for quasiunipotent, and $u$ for unipotent. We use standard notations from the Iskovskikh classification of three-dimensional Fano varieties (see [3]) and $X_{4}$ is a smooth three-dimensional quartic.

The Fano threefolds in Table 4 are combined horizontally in the following way. The LG model of the one on the left is a quotient of the LG model of the one on the right. Taking these quotients makes the monodromy of the fiber over 0 quasiunipotent and creates deeper singularities.

Table 4 gives a new approach to the celebrated Iskovskikh-Manin counterexample to the Lüroth problem [4]-nonrationality of $X_{4}$. Similar considerations and the assumption of HMS imply that all smooth Fano threefolds $V_{10}$ are nonrational. The proof of Theorem 3.3 is based on the fact that the monodromy at the zero fiber of the Landau-Ginzburg model corresponds to the Serre functor of the Fukaya-Seidel category of this fiber. We give a brief argument explaining the use of the Serre functor for studying nonrationality questions at the end of Section 4.

Questions 3.1. Does a similar correspondence hold in higher dimensions?

Is it possible to have a Serre functor of a singular fiber behaving as a Serre functor of a smooth variety and still have nonrationality?

Is it possible to have a nonunipotent monodromy and still have rationality? What other criteria do we apply then?

We discuss an answer to these questions in the next section.

\section{THE FOUR-DIMENSIONAL CUBIC}

In order to study the above questions, we need to introduce a new technique - the superschemes and sheaves of vanishing cycles associated with them.

Definition 4.1. The scheme defined as

$$
\operatorname{Proj}\left(\mathbb{C}\left[x_{0}, \ldots, x_{n}\right] \otimes \bigwedge \mathbb{C}\left[\eta_{1}^{1}, \ldots, \eta_{k_{1}}^{1}\right] \otimes \ldots \otimes \bigwedge \mathbb{C}\left[\eta_{1}^{m}, \ldots, \eta_{k_{m}}^{m}\right]\right),
$$

$n, k_{1}, \ldots, k_{m} \in \mathbb{Z}_{>0}$, is called a superscheme.

Here $\bigwedge \mathbb{C}\left[\eta_{1}^{i}, \ldots, \eta_{k_{i}}^{i}\right]$ denotes the exterior algebra. Let us consider the following two log schemes [5]: $P=\left(\mathrm{pt}, S^{1} \times \mathbb{R}_{\geq 0}\right)$ and $i: Y_{0} \rightarrow Y_{/ \mathbb{A}}$, where $Y_{0}$ is any singular fiber of the LandauGinzburg model for $X$.

Definition 4.2. We call the stack $\mathrm{KSS}=\operatorname{Maps}\left(P_{/ \mathbb{A}}, Y_{/ \mathbb{A}}\right)$ a Kato stratified space.

Here $Y_{/ \mathbb{A}}$ relates to the perverse sheaf of vanishing cycles on the Landau-Ginzburg model and $P$ measures the monodromy in different strata of $\operatorname{KSS}=\operatorname{Maps}\left(P_{/ \mathbb{A}}, Y_{/ \mathbb{A}}\right)$. The Kato stratified

\footnotetext{
${ }^{1}$ Here we consider the monodromy action on the second cohomology group of the general fiber.
} 


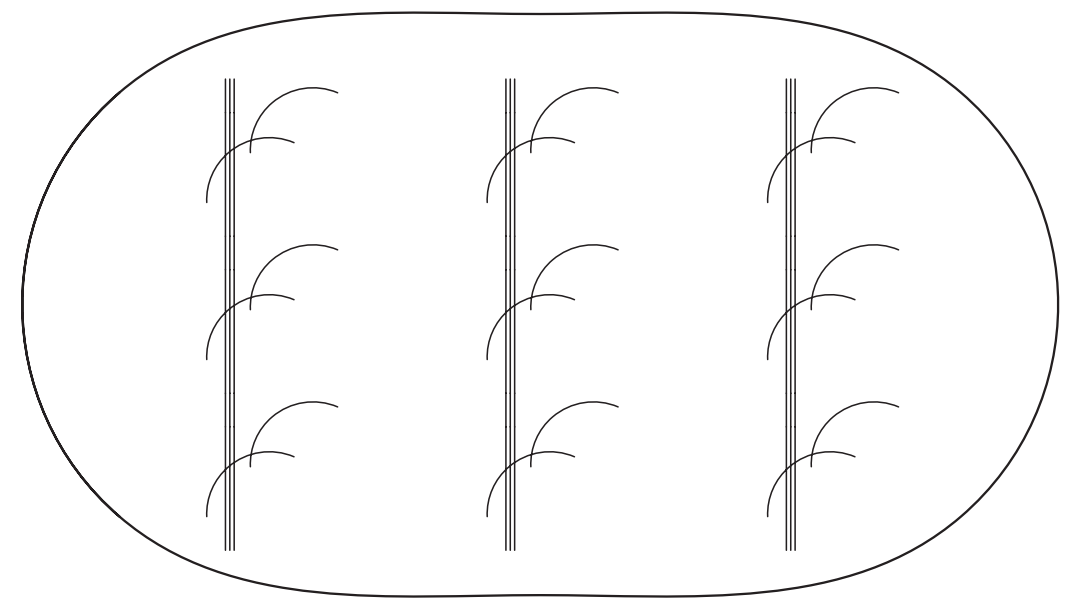

Fig. 2. Singularities for the four-dimensional cubic.

space has a noncommutative mixed Hodge structure on its hypercohomology [6]. (For more details see our forthcoming paper "Homological Mirror Symmetry, Monodromies and Cycles" and "Mirror Symmetry and Vanishing Cycles" by Gross and Katzarkov.)

We will apply the above general definitions to a classical example. We describe the LandauGinzburg model of the four-dimensional cubic. More precisely, we study the singularities of the fiber over 0 of this Landau-Ginzburg model (they can be seen in Fig. 2). It is an elliptic surface $S$ with three singular fibers; all of them are of type $\widetilde{E}_{6}$.

We describe the picture of Fig. 2 algebraically following [10]. The (noncompact) LandauGinzburg model for the four-dimensional cubic is

$$
\left\{f:=\frac{\left(x_{1}+x_{2}+1\right)^{3}}{x_{1} x_{2} y_{1} y_{2}}+y_{1}+y_{2}=0\right\} \subset \operatorname{Spec} \mathbb{C}\left[x_{1}, x_{1}^{-1}, x_{2}, x_{2}^{-1}, y_{1}, y_{1}^{-1}, y_{2}, y_{2}^{-1}\right]
$$

Compactify the pencil $\left\{f+\lambda=0, \lambda \in \mathbb{A}^{1}\right\}$ near $\lambda=0$ in the product of projective spaces:

$$
\begin{aligned}
F^{\text {sing }} & =\left\{y_{0}^{3}\left(x_{1}+x_{2}+x_{3}\right)^{3}+y_{1} y_{2}\left(\lambda y_{0}+y_{1}+y_{2}\right) x_{1} x_{2} x_{3}=0\right\} \\
& \subset \mathbb{P}\left(y_{0}: y_{1}: y_{2}\right) \times \mathbb{P}\left(x_{1}: x_{2}: x_{3}\right) \times \mathbb{A}(\lambda)
\end{aligned}
$$

We resolve the singularities of the general fiber $F_{\lambda}^{\text {sing }}, \lambda \neq 0$. Its singularities are the union of lines

$$
l_{0}^{1, \lambda}, l_{0}^{2, \lambda}, l_{1}^{\lambda}, l_{2}^{\lambda}, l_{3}^{\lambda}, s_{1}^{\lambda}, s_{2}^{\lambda}, s_{3}^{\lambda}, s_{4}^{\lambda}, s_{5}^{\lambda}, s_{6}^{\lambda}, s_{7}^{\lambda}, s_{8}^{\lambda}, s_{9}^{\lambda}, s_{10}^{\lambda}, s_{11}^{\lambda}, s_{12}^{\lambda}, s_{13}^{\lambda}, s_{14}^{\lambda}, s_{15}^{\lambda}, s_{16}^{\lambda}, s_{17}^{\lambda}, s_{18}^{\lambda}, s_{19}^{\lambda}
$$

in the fiber over $\lambda$, that is, on a hypersurface in $\mathbb{P}\left(x_{1}: x_{2}: x_{3}\right) \times \mathbb{P}\left(y_{0}: y_{1}: y_{2}\right)$.

These singularities are "horizontal" everywhere except for the fiber over $\lambda=0$, where the lines $l_{0}^{1,0}, l_{0}^{2,0}$, and $s_{19}^{0}$ coincide. After blowing up these coinciding lines, we get a fourfold $Y$. The fiber over $\lambda=0$ after the blow-up is the union of the proper image of the fiber of $F^{\text {sing }}$ over 0 (we denote it by $\left.E_{0}\right)$ and the exceptional divisor $E$. Let $S=E_{0} \cap E$. All singularities of $Y$ in the neighborhood of $S$ are "horizontal" (in particular, after blowing up $\ell=l_{0}^{1,0}=l_{0}^{2,0}=s_{19}^{0}$ it becomes three lines lying on $E$, which we denote by $l_{0}^{1,0}, l_{0}^{2,0}$, and $s_{19}^{0}$ ); that is, there is their resolution such that the exceptional divisor does not have any component in the fiber of $Y \rightarrow \mathbb{A}^{1}$ in the neighborhood of $\lambda=0$. So the singular set of the fiber of the resolution of $Y$ over 0 is $E_{0} \cap E=S$.

Now blow up $\ell$. It is given by

$$
x_{1}+x_{2}+x_{3}=y_{1}=y_{2}=\lambda=0 .
$$


Let $a=x_{1}+x_{2}+x_{3}$. Change the variables (using $a$ instead of $x_{3}$ ). The line $\ell$ lies in two similar local charts $x_{1} \neq 0, y_{0} \neq 0$ and $x_{2} \neq 0, y_{0} \neq 0$. Consider, for instance, the second one. That is, put $x_{2}=y_{0}=1$. Denote $x_{1}$ by $x$ for simplicity. We get the hypersurface

$$
\left\{a^{3}+x(a-x-1) y_{1} y_{2}\left(\lambda+y_{1}+y_{2}\right)\right\} \subset \mathbb{A}\left(a, x, y_{1}, y_{2}, \lambda\right)
$$

and need to blow up the line

$$
\left\{a=y_{1}=y_{2}=\lambda=0\right\}
$$

(i.e., the axis $x$ ). We have four standard local charts in the blow-up. More particular, the blow-up along the axis $x$ is given by the same equation in

$$
\mathbb{A}\left(a, x, y_{1}, y_{2}, \lambda\right) \times \mathbb{P}\left(a^{\prime}: x^{\prime}: y_{1}^{\prime}: y_{2}^{\prime}, \lambda^{\prime}\right)
$$

intersected with

$$
\left\{a^{\prime} y_{1}=a y_{1}^{\prime}, a^{\prime} y_{2}=a y_{2}^{\prime}, a^{\prime} \lambda=a \lambda^{\prime}, y_{1}^{\prime} y_{2}=y_{1} y_{2}^{\prime}, y_{1}^{\prime} \lambda=y_{1} \lambda^{\prime}, y_{2}^{\prime} \lambda=y_{2} \lambda^{\prime}\right\}
$$

The local charts of the blow-up are $a^{\prime} \neq 0, y_{1}^{\prime} \neq 0, y_{2}^{\prime} \neq 0$, and $\lambda^{\prime} \neq 0$. In these local charts we write $a, y_{1}, y_{2}$, and $\lambda$ instead of $a^{\prime}, y_{1}^{\prime}, y_{2}^{\prime}$, and $\lambda^{\prime}$ for simplicity; actually, the equation of the blown-up hypersurface, say, in the first local chart is obtained from the initial equation by a change of coordinates

$$
a \mapsto a, \quad y_{1} \mapsto y_{1} a, \quad y_{2} \mapsto y_{2} a, \quad \lambda \mapsto \lambda a
$$

and division by a power of $a$. The exceptional set is given by $a=0$. So we use the notation $a \neq 0$ for this local chart and consider the equation of the blow-up described above.

The local chart $a \neq 0$. The hypersurface is

$$
\left\{1+x(a-x-1) y_{1} y_{2}\left(\lambda+y_{1}+y_{2}\right)=0\right\} \subset \mathbb{A}\left(a, x, y_{1}, y_{2}, \lambda\right) ;
$$

it is smooth. The exceptional set is

$$
\left\{1-x(x+1) y_{1} y_{2}\left(\lambda+y_{1}+y_{2}\right)=0\right\} \subset \mathbb{A}\left(a, x, y_{1}, y_{2}, \lambda\right) .
$$

The surface $S$, given by the intersection of the exceptional set with $E_{0}=\{\lambda=0\}$, is

$$
\left\{1-x(x+1) y_{1} y_{2}\left(y_{1}+y_{2}\right)=0\right\} \subset \mathbb{A}\left(x, y_{1}, y_{2}\right) .
$$

So it is a fibration by smooth affine curves of degree 3 over the axis $x$.

The local chart $y_{1} \neq 0$. The hypersurface is

$$
\left\{a^{3}+x\left(y_{1} a-x-1\right) y_{2}\left(\lambda+y_{2}+1\right)=0\right\} \subset \mathbb{A}\left(a, x, y_{1}, y_{2}, \lambda\right) .
$$

The exceptional set is

$$
\left\{a^{3}-x(x+1) y_{2}\left(\lambda+y_{2}+1\right)=0\right\} \subset \mathbb{A}\left(a, x, y_{1}, y_{2}, \lambda\right) .
$$

The singularities are proper transforms of $s_{13}, l_{1}, s_{14}, l_{2}$, and $l_{0}^{1}$. The surface $S$ is

$$
\left\{a^{3}-x(x+1) y_{2}\left(y_{2}+1\right)=0\right\} \subset \mathbb{A}\left(a, x, y_{2}\right) .
$$

So it is a fibration by smooth affine curves of degree 3 over the axis $x$ for $x \neq 0,-1$; the fibers over $x=0,-1$ are triple lines. Its singularities are four singular points of type $A_{2}$; they are intersection points of $S$ with the singularities of $Y$. 
The local chart $y_{2} \neq 0$. It is similar to the previous one.

The local chart $\lambda \neq 0$. The surface $S$ does not lie in this local chart, so we will not analyze it.

Gluing together these local charts, we get an (affine) elliptic surface with two triple lines and three singular points lying on each of these lines (the intersections with $l_{1}, l_{2}, l_{0}^{1}, l_{0}^{2}, s_{13}, s_{14}, s_{16}$, and $\left.s_{17}\right)$. The images of these lines on $F^{\text {sing }}$ are the points $(0: 1:-1) \times(1: 0: 0) \times(0)$ and $(-1: 1: 0) \times(1: 0: 0) \times(0)$. There is the same triple line in the local chart $x_{1} \neq 0, y_{0} \neq 0$ lying over $(1: 0:-1) \times(1: 0: 0) \times(0)$. Hence $S$ is an elliptic surface with three triple lines and three singular points of type $A_{2}$ lying on each of them.

It is easy to see that the singularities are "horizontal" now in the neighborhood of $S$ (their lower terms do not depend on parameters). We blow up one of them, say, $s_{13}$ in the local chart $y_{1} \neq 0$ for example.

We need to blow up the line

$$
\left\{a=x=y_{2}=0\right\}
$$

on

$$
\left\{a^{3}+x\left(a y_{1}-x-1\right) y_{2}\left(\lambda+y_{2}+1\right)=0\right\} .
$$

As usual, consider local charts.

The local chart $a \neq 0$. The hypersurface is

$$
\left\{a+x\left(a y_{1}-a x-1\right) y_{2}\left(\lambda+a y_{2}+1\right)=0\right\} \subset \mathbb{A}\left(a, x, y_{1}, y_{2}, \lambda\right) ;
$$

it is smooth. The exceptional set is the union of

$$
E_{1}^{a}=\{a=x=0\}, \quad E_{2}^{a}=\left\{a=y_{2}=0\right\}, \quad E_{3}^{a}=\{a=\lambda+1=0\} .
$$

The surface $S$, given by $\lambda=0$, is smooth in this local chart. The intersections of exceptional divisors with $S$ are two lines.

The local chart $x \neq 0$. The hypersurface is

$$
\left\{x a^{3}+\left(y_{1} a x-x-1\right) y_{2}\left(\lambda+x y_{2}+1\right)=0\right\} \subset \mathbb{A}\left(a, x, y_{1}, y_{2}, \lambda\right) .
$$

The exceptional set is the union of

$$
E_{1}^{x}=\left\{x=y_{2}=0\right\}=E_{2}^{a}, \quad E_{2}^{x}=\{x=\lambda+1=0\}=E_{3}^{a} .
$$

All singularities are proper transforms of ones before this blow-up.

The local chart $y_{2} \neq 0$. The hypersurface is

$$
\left\{y_{2} a^{3}+x\left(a y_{1} y_{2}-x y_{2}-1\right)\left(\lambda+y_{2}+1\right)=0\right\} \subset \mathbb{A}\left(a, x, y_{1}, y_{2}, \lambda\right) .
$$

The exceptional set is the union of

$$
E_{1}^{y_{2}}=\left\{x=y_{2}=0\right\}=E_{1}^{a}, \quad E_{2}^{y_{2}}=\left\{y_{2}=\lambda+1=0\right\}=E_{3}^{a} .
$$

All singularities are proper transforms of ones before this blow-up. There is a proper transform of the triple fiber; it intersects the proper transform of $S$ (i.e., the blow-up of $S$ at a point of type $A_{2}$ ) at one point.

Finally, after a resolution in the neighborhood of $S$ we get an elliptic surface over $\mathbb{P}^{1}$ with three fibers of type $\widetilde{E}_{6}$.

In light of [8] and [11] the results of the above calculation are not surprising at all. We have

Theorem 4.1. The singular set of the fiber over 0 of the Landau-Ginzburg model of the fourdimensional cubic is a K3 surface. 
Table 5. Mirror Symmetry for superschemes

\begin{tabular}{|c|c|}
\hline Superschemes & Kato stratified spaces \\
\hline $\begin{array}{l}F+z^{2}+\eta_{1} \eta_{2}+\eta_{3} \eta_{4}=0, \\
\text { where } F \text { is a general cubic polynomial } \\
\text { in projective variables } x_{0}, \ldots, x_{5}\end{array}$ \\
\end{tabular}

The above calculation suggests

Conjecture 4.1. The generic four-dimensional cubic is not rational.

We outline a brief explanation of the connection of rationality questions with monodromy invariants. This connection will assume HMS.

1. The category $\lim _{D_{i}} D_{\text {compact }}^{\mathrm{b}}\left(X \backslash D_{i}\right)$ is a birational invariant.

2. According to HMS (Table 2), the category $\lim _{Y_{i}} \operatorname{FS}\left(Y_{i}\right)$ is a birational invariant too.

In [7] we introduce a deeper invariant-the monodromy of $\mathcal{F}$, the perverse sheaf of vanishing cycles associated with the Landau-Ginzburg model. This monodromy is recorded by the mixed Hodge structure associated with the Kato stratified space.

3. The generalized HMS discussed in Section 2 suggests that if the monodromy of $\mathcal{F}$ on one of the singular fibers with nonisolated singularities is nontrivial, it stays nontrivial for the limit Landau-Ginzburg model and for the limit category $\lim _{Y_{i}} \operatorname{FS}\left(Y_{i}\right)$.

4. As Theorem 3.2 suggests, the monodromy of the limit Landau-Ginzburg model of a rational manifold is trivial.

We return now to Conjecture 4.1. The calculation in this section shows the following:

1. The Serre functor of the Fukaya-Seidel category of the fiber at 0 is a shift by 2 for the Landau-Ginzburg model for any four-dimensional cubic, so the Serre functor behaves as the Serre functor of a smooth compact algebraic commutative K3 surface.

2. In the case of a generic four-dimensional cubic the monodromy of $\mathcal{F}$ for the limit LandauGinzburg model is nontrivial. This can be seen as follows. In [7] we show a correspondence between superschemes and Kato stratified spaces. In the case of a generic four-dimensional cubic the nontriviality of the monodromy can be seen on the supercurve described in Table 5.

As follows from [1], the rationality implies that all Kato stratified spaces associated with the supercurves of the four-dimensional cubic should be mirrors of usual curves.

These observations give a negative answer to the questions posed in the previous section and give some strong validation of Conjecture 4.1. More details will appear elsewhere.

\section{ACKNOWLEDGMENTS}

We are grateful to D. Auroux, V. Golyshev, M. Gross, M. Kontsevich, A. Kuznetsov, D. Orlov, T. Pantev, P. Seidel, K. Shramov, and D. Stepanov for many useful conversations. Many thanks go to V. Boutchaktchiev, without whom this paper would not have been written. We are grateful to IHES, ESI, and EPFL for the support.

The first author was partially supported by the NSF (project no. DMS0600800), NSF FRG (project no. DMS-0652633), FWF (project no. P20778), and an ERC grant. The second author 
was partially supported by the FWF (project no. P20778), Russian Foundation for Basic Research (project nos. 08-01-00395-a and 06-01-72017-MNTI-a), and a grant of the President of the Russian Federation (project no. NSh-1987.2008.1).

\section{REFERENCES}

1. M. Abouzaid, D. Auroux, and L. Katzarkov, "Homological Mirror Symmetry for Blowups," Preprint (Massachusetts Inst. Technol., 2008).

2. V. V. Golyshev, "Classification Problems and Mirror Duality," in Surveys in Geometry and Number Theory, Ed. by N. Young (Cambridge Univ. Press, Cambridge, 2007), LMS Lect. Note Ser. 338, pp. 88-121; arXiv: math/0510287.

3. V. A. Iskovskikh, "Fano 3-folds. I," Izv. Akad. Nauk SSSR, Ser. Mat. 41 (3), 516-562 (1977) [Math. USSR, Izv. 11, 485-527 (1977)]; "Fano 3-folds. II," Izv. Akad. Nauk SSSR, Ser. Mat. 42 (3), 506-549 (1978) [Math. USSR, Izv. 12, 469-506 (1978)].

4. V. A. Iskovskikh and Yu. I. Manin, "Three-Dimensional Quartics and Counterexamples to the Lüroth Problem," Mat. Sb. 86 (1), 140-166 (1971) [Math. USSR, Sb. 15, 141-166 (1971)].

5. K. Kato and Ch. Nakayama, "Log Betti Cohomology, Log Étale Cohomology, and Log de Rham Cohomology of Log Schemes over $\mathbb{C}, "$ Kodai Math. J. 22 (2), 161-186 (1999).

6. L. Katzarkov, M. Kontsevich, and T. Pantev, "Hodge Theoretic Aspects of Mirror Symmetry," arXiv: 0806.0107.

7. L. Katzarkov and V. Przyjalkowski, "Generalized Homological Mirror Symmetry, Perverse Sheaves and Questions of Rationality," in Topology of Stratified Spaces, Berkeley, MSRI, Sept. 2008, Ed. by F. Bogomolov, A. Libgober, and S. Cappell (Cambridge Univ. Press, Cambridge, 2009), Math. Sci. Res. Inst. Publ. (in press).

8. C. Leung, "Geometric Aspects of Mirror Symmetry (with SYZ for Rigid CY Manifolds)," Proc. Int. Congr. Chin. Math. 2001; arXiv: math/0204168.

9. D. O. Orlov, "Projective Bundles, Monoidal Transformations, and Derived Categories of Coherent Sheaves," Izv. Ross. Akad. Nauk, Ser. Mat. 56 (4), 852-862 (1992) [Russ. Acad. Sci., Izv. Math. 41 (1), 133-141 (1993)].

10. V. Przyjalkowski, "On Landau-Ginzburg Models for Fano Varieties," Commun. Number Theory Phys. 1 (4), 713-728 (2008); arXiv: 0707.3758.

11. S. Sethi, "Supermanifolds, Rigid Manifolds and Mirror Symmetry," Nucl. Phys. B 430, 31-50 (1994); arXiv: hep-th/9404186.

This article was submitted by the authors in English 